\title{
Irreproducible results
}

\section{from Robert M. May}

CONSIDER the following game, played with the combinatorial theorist's mythical urn containing black and white balls. Initially the urn contains only two balls, one black and one white. Choose one ball at random from the urn, note its colour, and then replace it plus a new ball of the same colour as the chosen ball. The game thus proceeds, each round consisting of the random selection of one ball from the urn and replacement of this ball together with a new ball of the same colour. After $N$ such rounds, the urn will contain a total of $N+2$ balls.

After many rounds, the urn will contain a large number of balls. What fraction of the balls will be white? The reader is invited to pause here, and to make a determined effort to guess the answer.

The answer is that for large $N$ the proportion of white balls will tend to converge to some limiting value, $p$ say: but this limiting value $p$ is equally likely to take any value between 0 and 1. That is, when I play this game my plot of the proportion of white balls in the urn as a function of the number of rounds (or, equivalently, time) will exhibit some initial wiggles, but will eventually settle to a steady flat line corresponding to, say $p=0.391 \ldots$. When you play the game you see similar initial fluctuations, which damp out as the total number of balls gets large, and your experiment will similarly settle to some steady but different value of, say, $p=0.893 .$. , and so on. Each run of this experiment will yield to the experimenter an illusion of determinism, as his results settle to a steady limiting value; this value is, however, a random variable, differing from one experiment to the next, and determined largely by the statistical vagaries of the first few turns.

In short, this simple game provides an example where an underlying stochastic process gives results which appear deterministic (at least to the person who witnesses but one run). It is, in a sense, the mirror image of the phenomenon I recently reviewed (Nature, 261, 459-467; 1976) whereby simple deterministic processes can produce results which appear indistinguishable from random fluctuations.

The above game has been known to mathematicians for some time, and has been discussed in relation to the spread of infection (Eggenberger and Polya, Zeit. angew. Math. Mech., 3, 279-289; 1923) and to stochastic population growth (Blackwell and Kendall, J. appl. Prob., 1, 284-296; 1964), but the basic messages have tended to become buried under mathematical encrustations. Cohen (BioScience, 26, 391-394; 1976), in a paper exuberantly entitled "Irreproducible Results and the Breeding of Pigs (or Nondegenerate Limit Random Variables in Biology)", has disinterred the phenomenon, and suggested that it may have widespread relevance in population biology.

Geneticists such as Robertson (Proc. R. Soc. Lond., B153, 234-249; 1960) and Hill (Biometrics, 30, 363-366; 1974) have realised that this urn-andball game captures some of the essential features of plant and animal breeding programmes, and that replicate lines from the same initial population can be very different in the limit they reach. Cohen suggests that similar "nondegenerate limits" may arise in many ecological contexts. For example, differences in successional sequences and in climax states in apparently similar habitats may not be due to causal differences between habtitats, but may arise as variations in an ensemble of habitats (ultimately dependent on the statistical accidents of early colonisation). Differences in "species composition on apparently similar islands may result from the operation of identical forces which produce regularity only in an ensemble of islands. Differences in the sizes of prides of lions or in the social organisation of troops of Japanese macaques may reflect the inherently but lawfully variable outcome of identical underlying forces, rather than deterministic

\section{Bibliography of plant genetic resources}

DURING the replacement of traditional crops by new varieties a great deal of the potential genetic variation present in the old varieties and their wild relatives has been lost. Over the past two decades however, interest in the conservation and use of the plant genetic resources that remain has been rapidly growing. The literature on the subject has naturally grown with it, and the first extensive bibliography of work in plant genetic resources has just been produced. It has been compiled by J. G. Hawkes, J. T. Williams and Jean Hanson of the University of Birmingham and as well as covering the literature devoted specifically to crop genetic resources, there is also extensive coverage of crop origins, variation and evolution, along with appropriate references in ecology, genetics, practical agriculture, phytopathology, and entomology.

The bibliography is produced by the International Board for Plant Genetic Resources and can be obtained from FAO, Rome. ecological differences". Most of these suggestions have been made previously by other people, but not in so precise a form.

Turning to behaviour, Cohen suggests that it is "possible that some of the significant differences among mother-child interactions, which are obvious by the time a child reaches five years of age, are due neither to inherent differences among individuals nor to environmental differences, but to sequentially dependent random forces applying equally to all motherinfant pairs".

Cohen concludes on a metaphysical note. If we had been born in another universe which had started from exactly the same initial conditions as our present one and which had been subject to the same dynamics, would we necessarily infer the same laws of nature as we infer for this universe? The urn-and-ball game holds the suggestion that our present Universe may be an irreproducible result.

\section{Human trypanosomiasis today \\ from F. E. G. Cox}

TRYPANOSOMIASIS has received a considerable amount of attention recently (see Nature, 249, 677; 1974; 262, 85; 1976 and the Pan American Health Organisation Symposium on New Approaches to American Trypanosomiasis, 1975) and the latest number of the Transactions of the Royal Society of Tropical Medicine and Hygiene (70 part 2, 1976) contains a refreshingly summarised statement of the current status of this group of diseases under the title "Trypanosomiasis Today". Missing are the results of biochemical, ultrastructural and immunological studies that have made this field so attractive to many scientists over the past few years and instead there is basic information emphasising the need for sustained effort to be applied in the field and in the pharmaceutical laboratory.

In Africa, human trypanosomiasis, or sleeping sickness, is still widespread in the tsetse fly-infested areas where it exists in the form of unstable "controlled endemicity" (de Raadt, Trans. R. Soc. trop. Med. Hyg., 70, 114; 1976). Even in West Africa where the disease is under control in many places the number of new cases is increasing by about 10 per 100,000 people and there were 2,511 recorded new cases in Africa in 1974. This is a vast improvement on the 1970 figures of 9,140 but trypanosomiasis is still prevalent and 